\title{
Virtual Reality (VR) Technology and Landscape Architecture
}

\author{
Jialu Song, Sijia Huang \\ Sichuan Agricultural University
}

Keywords: Virtual Reality (VR); Landscape architecture

\begin{abstract}
Virtual reality technology has been applied to a variety of fields. Various kinds of organizations have been deepening the study of this technology. This article is a discourse of the application of the VR technology in landscape design in the field of landscape architecture. The connection between virtual reality technology and landscape scene construction innovates the traditional design process and realizes the interaction between designers and customers and the public in the design process
\end{abstract}

\section{The concept and developments of VR technology}

\subsection{The concept of VR technology}

VR stands for Virtual Reality, which was proposed by Jaron Lanier, founder of VPL Research Company in 1989. It has a history of more than 20 years. The technology works by creating a computer system with multiple senses of sight, hearing, touch, and so on. Users immerse themselves in the system of virtual environment with various hardware devices and interact with the virtual environment, thus causing real time changes of virtual environment.

"Virtual reality is a computer system that can create and experience the "Virtual World". A Virtual world is the whole of a Virtual Environment or a given simulation object. The virtual environment is generated by the computer, and the interactive visual scene simulation of immersive feeling is made by the interaction of visual, auditory and tactile effects on users. ${ }^{[1]}$

\subsection{The development of VR technology}

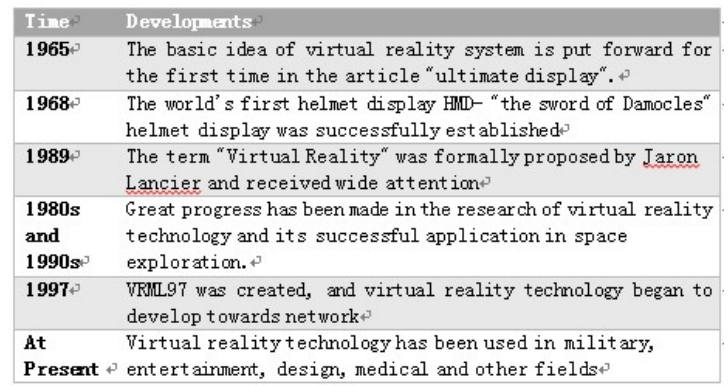

(Figure 1 Source: Self making)

\section{The application of VR technology in China}

At present, although the research on virtual reality technology in China is still far behind the developed countries, the technology has received wide attention in recent decade. Many domestic research institutions are carrying out the related application of virtual reality technology and have achieved some positive results. For example, the National Compact Disc Engineering Research Center of Tsinghua University has realized the large-scale virtual scenes of Potala Palace by using virtual reality technology.

With the deepening of the research on virtual reality technology in China, the technology has been gradually applied to many fields, such as entertainment, military, production and manufacturing, the ancient cultural relics restoration, urban planning, architectural design, landscape design, interior design, etc. The wide application of virtual reality technology is undoubtedly an important opportunity for the growth and renovation of many fields.

In modern landscape design, landscape scene plays an important role in presenting spatial relationship, landscape structure and node sequence. VR technology can make the construction of landscape scene more effective. An immersive virtual environment system is a large integrated multi-level environment created by computer graphics, image processing and pattern recognition, intelligent interface technology, artificial intelligence technology, sensor technology, speech processing and audio and video technology, network technology, parallel processing technology and high-performance computer systems. Therefore, the virtual reality technology is a highly integrated 
information technology. ${ }^{[2]}$ It is undoubtedly beneficial to apply this highly specialized integrated technology to the construction of modern landscape design scenes.

\section{VR technology and landscape architecture}

\subsection{The application of VR technology in landscape scene}

\subsubsection{Create an immersive sense of "Being There"}

One of the important features of VR technology is to create the immersive feeling of "being in the real world". Through the interaction between users and computers, users can truly experience the existence of virtual environment. This feature of VR technology is widely used in landscape design. The virtual reality technology is used to construct the landscape scene directly on the computer system. Then through the relevant hardware output equipment, users can achieve the effect of as if they are in the landscape scene. Visually and effectively feel the unconstructed landscape design and offer comments and feedbacks. The application of VR technology in landscape scene construction adds users' experience of uncompleted landscape design, and creates a new way for designer to showcase the design to users.

\subsubsection{Interact with the environment}

The interaction of VR technology means that users interact with virtual things and objects around them as if they were in a real environment. In the whole interaction process, the user is the interaction subject, and the virtual things are the interaction objects. The interaction between subject and objects is omnidirectional.

For example, in constructing landscape design scene, one can set up certain landscaping devices with the temperature sensor, photosensitive sensor, pressure sensor and visual sensor in the energy convergence area of the landscape. The sensor would help create different landscape modes through the perception of different direction of energy (sun, breeze, temperature, etc.); This landscape scene attracted people, and energy changes resulting from the number of people will trigger the sensor for new changes and form a different landscape. The landscape will be able to reflect the invisible forces in a visible way. This interaction between people and the environment is achieved through VR devices. It helps users interact naturally with multidimensional virtual environments.

\subsubsection{Imagination motivates users to think creatively}

Because of the interactive and intuitive nature of VR technology, users are able to gain both the perceptual and rational understanding of the virtual environment, thus to inspire a series of creative thinking. This feature is especially useful for landscape design.
First of all, designers often encounter technical or idea bottlenecks in the design process. This problem arises partly because designers do not have a complete and intuitive scene construction and display for their landscape scenes, so their thinking is confined in some way. But with VR technology, designers can move the models to VR and directly access their own design of virtual landscape scenes, immersive intuitively feel themselves in a virtual environment design, so as to inspire creative thinking to improve the overall design.

Secondly, for customers, with the application of VR technology, customers can be exposed to the landscape scene by wearing the corresponding virtual reality equipment, so as to truly and effectively experience the whole landscape design. Break the traditional way of 2D or 3D computer display, and conduct feedback directly in VR during the comprehensive experience.

\subsubsection{Break through the bottleneck of traditional landscape design methods}

In landscape design, designers create landscape scenes through their own understanding of landscape, combined with their own relevant designing experience, and through the deployment of plants, layout of landscape items and other ways. Traditional landscape design is a difficult project to grasp, from the designer's construction of landscape scenes to the decision-makers' evaluation of design results and understanding of design intention. Through the use of VR technology designers, decision-makers, engineering construction personnels and the public can watch the effect of landscape design from any angle at any time, as it were, to feel the landscape design and understand the designing idea, and offer timely feedback, which would greatly strengthen the communication among different project teams and improve work efficiency, so as to break through the bottleneck of traditional landscape design method.

\subsection{The advantages of VR technology focus}

\subsubsection{Analysis of the spatial experience of VR technology in landscape design}

In landscape design, to achieve a unified dynamic spatial perception of the space constructed by landscape scenes, it is necessary to achieve the goal through continuous movement of users in landscape scenes. Traditionally, landscape designers would analyze and feel the space by building 3D models, drawing effect pictures of landscape scenes, and making animations of simulated landscape scenes. However, these methods are only based on users' vision and hearing, and have great limitations on the overall dynamic space experience. The real spatial experience for users should be a comprehensive spatial experience integrating visual, auditory, tactile and other behaviors. In the VR user experience, users can experience the visual effect space by adjusting their perspectives and movement in their virtual environment. With the help of VR technology, the construction of landscape scenes will be more authentic and rapid. 
Through the relevant VR equipment, users can get the most real and integrated spatial experience of landscape scenes, find out the advantages and disadvantages of its design and offer feedbacks. The application of virtual reality technology enables landscape designers to take into account different levels and design works more in line with the principle of "people-oriented".

\subsubsection{Analysis of physical environment of technology in landscape design}

In landscape design, the air flow direction and development intensity of the area can be regulated through the treatment of the terrain, thus affecting users' experience of the landscape environment. For example, slope topography can block the cool air flow, change the microclimate in the landscape area, and give users a more comfortable experience. If landscape design area is flat terrain, wind speed on the high side, can cause area high wind speed can cause environmental pollution, causing inconvenience to the user, will also impact on landscape design area of the surrounding environment, the temperature drop caused local area. Through the application of VR technology, landscape designers can simulate and construct landscape scenes, and simulate the real airflow environment of landscape design areas according to the environmental climate in the real world. Under the background of real environment data, the designer makes real-time dynamic modification to the terrain model in the landscape area. Designers of airflow direction and strength, which change due to topography, can experience in the virtual environment, so as to achieve the ideal effect.

In VR technology, the computer will measure the real-time airflow data, which is helpful for designer to optimize the original design, so that to modify the landscape in a more reasonable way, thus improving the micro-climate of the landscape.

\subsubsection{VR technology dynamically compares and edits elements in landscape design}

Dynamic comparison of design elements and real-time editing are the focuses of virtual reality technology. In landscape design, if the design elements can be compared and modified at any time, the requirements of landscape design can be more effectively met. The construction of landscape scene is an important part of landscape design. In the process of constructing landscape scene, designers need to consider the difference of landscape scenes. The application of virtual reality technology can meet the real-time switching of different landscape designs. With VR technology, designers can quickly and intuitively achieve the analysis and comparison of multiple designs, and directly switch virtual scenes of different designs.

After dynamic comparison, designers can also directly edit design elements through VR technology to realize the workflow of experience-compare-edit as a whole.

\subsection{The construction process of VR technology in landscape scene}

Since the advent of VRML technology in 1994, the research on virtual reality technology has been deepening continuously, and various virtual reality technologies have appeared successively. At present, VRML, Multigen Vega, Cult3D, Virtools, 360 panorama and domestic WEBMAX, 3DVRI, vr-platform and other virtual reality technologies are mainly available [3]. Because all of the operation of the VR Platform is based on a comprehensible way, the user does not need to master the complex programming code. The user can achieve visual reality through simple steps based on 3DS MAX model. Therefore, compared with other virtual reality technologies, VR-Platform technology can easily realize the application of virtual reality technology in landscape scene construction. Based on VR-Platform technology, the implementation process of virtual reality in landscape design has the following steps.

1. Use 3DS MAX to build model scenarios

Firstly, the landscape scene and each element model according to its own design scheme are built in 3DSMAX, and then the corresponding texture map is given to the model. Finally, add light source and required light for the whole landscape scene. The following points should be noted when establishing the virtual scene model:

(1) The size of the model scene should be consistent with the real size, and the measurement should not use $\mathrm{mm}$ as far as possible, otherwise the display effect in vr-plat-form will be affected due to excessive values.

(2) When establishing complex landscape scene topography, contour drawing can be carried out within 3DSMAX, and all contour lines can be combined into complex terrain models by using "mountain generation command".

(3) In the processing of model materials, attention should be paid to check the double-sided and material nesting, so as to ensure the overall fit of scene model materials and model.

(4) The completed scenario model is rigorously refined to minimize the number of faces in the model Remove the extra clutter and surfaces in the model to optimize the running speed of the virtual scene.

(5) When dealing with irregular objects such as rocks and plants, try to use "plane +TGA transparent texture". It can not only reduce the model pressure, but also achieve more real virtual effect. For animated objects that need to be processed into vr-platform editors for texture mapping, such as water bodies, fountains, etc., in 3DSMAX, they are only represented by plane.

(6) When establishing lighting, the effect requirements of all objects in the scene should be ensured. Artificial light source can be added to create the three-dimensional sense of objects in the scene, and the lighting is usually arranged in the way of "direct" + "sky".

\section{Processing of texture mapping}

In order to achieve the rendering effect of light and shadow in the later virtual reality scene, we need to use the baking function in 3DS MAX to render the texture. At the end of the processing, vrp-for-max plug-in is used to import the constructed landscape scene model into the vr-platform editor as a whole.

3.Edit the virtual reality scene. 
After the scenario model in 3DS MAX is imported into the vr-platform editor, the following actions are required.

(1) Turn on collision detection and set transparent barrier to the boundary. Avoid the phenomenon that the human passing walls and windows, falls into the dead corner, roams outside the scene and so on, which does not conform to the reality rule and affects the virtual experience.

(2) Add cameras that meet the corresponding roaming requirements, and set reasonable height and moving speed.

(3) The "bill board" setting is carried out for objects such as rocks and plants to meet the requirement that such objects always face the user.

(4) Add atx animation texture to waters, fountains and other materials to form corresponding simulation animations.

(5) Use sky box to add scene background.

(6) Add special effects such as fog effect or solar halo according to the display effect.

(7) Create the operating interface of virtual reality system such as buttons and navigation charts.

(8) Export and generate "EXE" format for native operation or "VRPIE" format file for network browsing.

4. Experience of virtual reality scenes

In addition to using the computer screen for browsing, one can also use the two-channel circular screen stereoscopic projection system to roam with 3D stereoscopic glasses [4], so as to achieve the immersive sense of virtual reality environment.

\subsection{The significance of application}

The application of VR technology in landscape design innovates the traditional design process and realizes the interaction between designers, customers and the public in the design process. Customers can roam in the virtual scenes at any time and anywhere, and give timely feedback on designing issues. The public can also participate in a lot of landscape design through this technology, and conduct dynamic experience on the uncompleted design, effectively avoiding the problem that "design does not meet the public demand" which occurs most frequently at present.

\section{Common problems of VR technology in landscape scene construction}

\subsection{Hardware resources that VR technology depends}

All the processes experienced in the application of virtual reality technology cannot be separated from the coordination of hardware and software. In landscape design, many points and surface elements are usually needed in the process of scene model construction. For example, the rough edges of the rocks, the ripples of the water, the exuberant plant leaves and so on will make the surface elements of the model reach millions levels. So the application of VR technology to landscape design requires high configuration computer hardware resources. This requirement has greatly constrained the penetration of VR technology in the field of landscape design.

\subsection{The highly priced equipment with poor experience}

With the continuous promotion of virtual reality technology, many hardware equipment companies of virtual reality technology have launched VR output devices such as helmets and glasses. However, these devices have poor experience, are slightly clumsy to wear, and also are relatively expensive for users. In terms of technology, the output device of data processing and related technology is not perfect, such as the loading speed and user movements cannot be matched, poor equipment pixels, dizziness after using devices, etc. All these factors have affected the promotion and application of virtual reality technology in the field of landscape design, hindering the support of users for this technology.

\subsection{The limitations of landscape design itself}

One of the major features of landscape design is that everyone has their own perceptual knowledge of this design, and there are multiple criteria for the evaluation of design. In fact, virtual reality technology can only construct landscape scenes as far as possible in terms of technology, and it cannot truly express the abstract connotation and aesthetic significance that designers inject into landscape scenes.

\subsection{The model database is not complete}

The incomplete model database is one of the factors that make many designers shy away from VR technology. The incomplete model database will seriously reduce the work efficiency of designers in the actual design, which directly affects the application of VR technology in the field of landscape design.

\section{The summary}

There are infinite opportunities to apply the VR technology to the field of landscape design. By using this technology, designers can quickly and visually construct landscape scenes for customers to experience. Although the current VR technology is not mature enough and many related problems need to be solved, I still believe that as long as the service providers of this technology continue to strengthen their technical research, designers will free their minds to use new technologies boldly. VR technology will be more widely used in landscape design in the near future to realize the innovation and transformation of design industry. 


\section{References}

1. Wei Youshuang, Yang Xianglong, Wang Fei. Virtual Reality and System Simulation [M]. Beijing: National Defense Industrial Press, 2004

2. Zhang jinzhao, Zhang Jindi, Zhang Jinrui. Virtual Reality 3D Network Programming Language VRML--Second Generation Network Programming Language [M]. Beijing: Tsinghua University Press, 2004
3. Zeng Junfeng, Ge Zuguo. Selection and Application of Virtual Reality Technology in Garden Design [J]. Journal of Changjiang University: Natural Science Edition, 2008, 5(4):25-28

4. Yan jun, Li Sumin, Zhong Jiong-guang. Research on Roaming System of Shanggantang Village Based on Virtual Reality Technology [J]. Architectural Science, 2008, (3):52-56 